\title{
A GREEN APPROACH FOR SYNTHESIS AND CHARACTERIZATION OF SILVER NANOPARTICLESAND THEIR ANTI-MICROBIAL ACTIVITY
}

\author{
M. Gopalakrishnan ${ }^{1} *$, A. Sheenu ${ }^{1}$, Binny $\operatorname{Varghese}^{1}$, J. Dharani ${ }^{1}$ \\ and S. Saranya ${ }^{1}$ \\ ${ }^{a}$ Department of Chemistry, Karpagam Academy of Higher Education, \\ Coimbatore-641 021, (Tamil Nadu) India. \\ *E-mail:gopalakrishnan.che22@gmail.com
}

\begin{abstract}
Silver nanoparticleswere synthesized using aqueous stem extract of Mirabilis jalapa as a reducing and stabilizing agent. This stem contains biomolecules such as alkaloids, flavonoids, triterpenes, steroid and phenolic compounds. The silver nanoparticles were characterized by UV-visible Spectroscopy (UV), Fourier Transform Infra-red Spectroscopy (FT-IR), powder X-ray diffraction (PXRD), Scanning Electron Microscope (SEM) and EDX analysis. A peak at $499 \mathrm{~nm}$ was obtained in UV-visible spectroscopy which indicates that the particle was polydispersed. PPXRD shows the crystalline nature of silver nanoparticles.Silver was used form long back as metallic silver, silver nitrate for the treatment of burns, wounds and many bacterial illnesses. The antimicrobial activities of silver nanoparticles were studied.
\end{abstract}

Keywords: Silver Nanoparticles, Mirabilisjalapa,Anti-Microbial

(C) RASĀYAN. All rights reserved

\section{INTRODUCTION}

Nanotechnology is one of the most active areas of research in modern material sciences. Nanotechnology has been used in many engineering fields such as electronics, mechanical, biomedical engineering Nanotechnology is a field, developing day by day, making an impact in all spheres of human life and creating a growing sense of excitement in the life sciences especially biomedical devices and biotechnology. Recently, the green chemistry which aims to reduce or eliminate substances hazardous to human health and the environment in the design, development and implementation of chemical processes and products is becoming more and more important ${ }^{1,2}$. To comply with the 12 principles of green chemistry, many types of research tried to avoid or reduce the uses of hazardous chemicals and solvents, such as using natural materials instead of toxic chemicals ${ }^{3-5}$. The utilization of nanoparticles is preferably having a significant role in this century. This is a challenging field of research with endless future prospects. There are two fundamental methodologies for the union of nanoparticles in particular top down and bottom up, some of them like strong state reaction, chemical reaction, co-precipitation and sol-gel manner and so on. Among all nanoparticles gold, copper, iron, palladium, zinc, quantum dots (CdS, $\mathrm{ZnS})$, Silver nanoparticles act a vital role in the numerous fields, especially in medical applications. Silver is a secure and efficient bactericidal metal since it is non-toxic to animal cells and extremely toxic to bacteria $^{6,7}$. The utilization of photochemical in the fusion of nanoparticles is significant beneficial interaction among nanotechnology and green chemistry ${ }^{8-10}$. The plant mediated green synthesis of nanoparticles is beneficial in light of the fact that it is earth Friendly and biocompatible, where it isn't important to utilize high pressure, energy, temperature and harmful chemicals ${ }^{11}$. The use of plants for the synthesis of nanoparticles is a rapid, low cost, eco-friendly, and single step method which can directly use for drug delivery and other similar application without any coating or core-shell techniques ${ }^{12}$. Mirabilis

Rasayan J. Chem., 12(3), 1072-1076(2019)

http://dx.doi.org/10.31788/RJC.2019.1235136

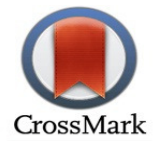


jalapa is a useful medicinal plant and has great importance in the field of ethno botany. A decoction of leaves is used against genitourinary system disorders while poultice of subterranean parts is used to treat injuries. It is also used for treating amenorrhea and dysmenorrhoea in women. Leaves of Mirabilis jalapa are applied on external wounds until recovery. Juice of leaves is mixed with water and use for the treatment of jaundice. A paste of leaves has emollient property ${ }^{13}$.In recent years, the green synthesis of metal nanoparticles has become a major focus of scientists. There are several reports on the synthesis of AgNPs from plants.

\section{Materials}

\section{MATERIALS AND METHODS}

Materials with high-grade AR silver nitrate was purchased from Sigma-Aldrich, India, throughout the reaction Double distilled water were used. Whatman no.1 filter papers were used for filtration. All glass wares used were washed well and dried using hot air oven.

\section{Preparation of leaf extract and Synthesis of AgNPs}

For extraction, Mirabilis jalapastem was gathered from Tirupur, Tamilnadu India. The gathered stem was cleaned well with distilled water. The stem is allowed to dry in shadow for 10 days. The dried stem is ground to fine powders and $2.5 \mathrm{~g}$ of powder is blended with $75 \mathrm{ml}$ of distilled water then it is boiled to $60^{\circ} \mathrm{C}$ for $10 \mathrm{~min}$. Subsequent to chilling off to ordinary room temperature, the concentrate was separated through normal filter paper to get free from powder and again sifted utilizing whatman channel paper to get clear stem to remove.The prepared silver nanoparticle was established using a UV-visible spectrum with a range of 300-700nm was recorded on a UV-visible JascoV-550 spectrophotometer. FT-IR measurements were utilized to decide biomolecules present in silver nanoparticles. The Ag nanoparticles were centrifuged at 9000rpm for 30min, Jusco 5300 model with the range of 400- 4000cm-1. SEM analysis was utilized to distinguish surface morphology. The blended AgNPs was dissipated onto a clean glass slide and covered the left to dry totally at room temperature. SEM examination was finished utilizing Supra Zeiss with a resolution of $1 \mathrm{~nm}$ at $30 \mathrm{kV}$ with $20 \mathrm{~mm}$ Oxford EDS detector. Dynamic light scattering (DLS) was utilized for estimation of normal hydrodynamic distances across and polydispersity files (PDIs) of the particles, Malvern Zetasizer Nano-ZS, Malvern Instruments, UK. Sample which is analyzed in triplicate at $20^{\circ} \mathrm{C}$ at a scattering angle of $173^{\circ}$ and double distilled water is utilized as a reference dispersing medium.

\section{Antimicrobial Activity}

The antimicrobial activity was done on various pathogenic bacteria. The test organisms utilized were Escherichia coli and Staphylococcus aureus. The agar well dissemination technique was utilized to decide the growth restraint. The sterile Muller Hinton Agar was arranged and poured in sterile Petri dishes and permitted to set. With the assistance of a clean well shaper, $6 \mathrm{~mm}$ breadth wells were punctured with uniform separating for different fixations for every concentrate. The log stage culture juices were taken and swabbed over the plate utilizing a sterile cotton swab to acquire uniform yard of culture. The plant

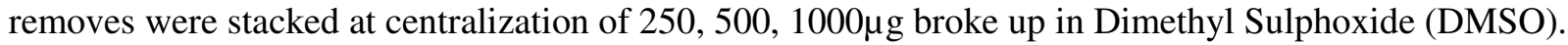

\section{UV-Visible Spectroscopy}

\section{RESULTS AND DISCUSSION}

The synthesized silver nanoparticles were characterized by using UV-Vis spectrophotometer. Absorption spectra of silver nanoparticles in the reaction mixture were recorded. The broadening of maximum adsorption peak at $449 \mathrm{~nm}$ indicates that the particles were polydispersed. The frequency and width of the surface Plasmon absorption depend on the size and shape of the metal nanoparticles as well as on the dielectric constant of the metal itself and its surrounding medium.

\section{Fourier Transforms Infra-Red Spectroscopy}

The FT-IR spectrum of the silver nanoparticles synthesized by the greener synthesis which was required in the range of $400-4000 \mathrm{~cm}^{-1}$. The presence of some functional groups as revealed by FTIR spectral analysis is showed. FTIR analysis of silver nanoparticles was also conducted to investigate the possible 
functional groups of biomolecules involved in synthesis and stabilization of silver nanoparticles. According to the FTIR transmittance peak of bio-functionalized silver nanoparticles synthesized from stem extract of Mirabilis jalapa showed transmittance peak at $3323 \mathrm{~cm}^{-1}$ which corresponds to $\mathrm{OH}$ stretching vibration. The synthesized silver nanoparticle also showed transmittance peak at $1620 \mathrm{~cm}^{-1}$ corresponds to $\mathrm{C}=\mathrm{O}$ ketones.

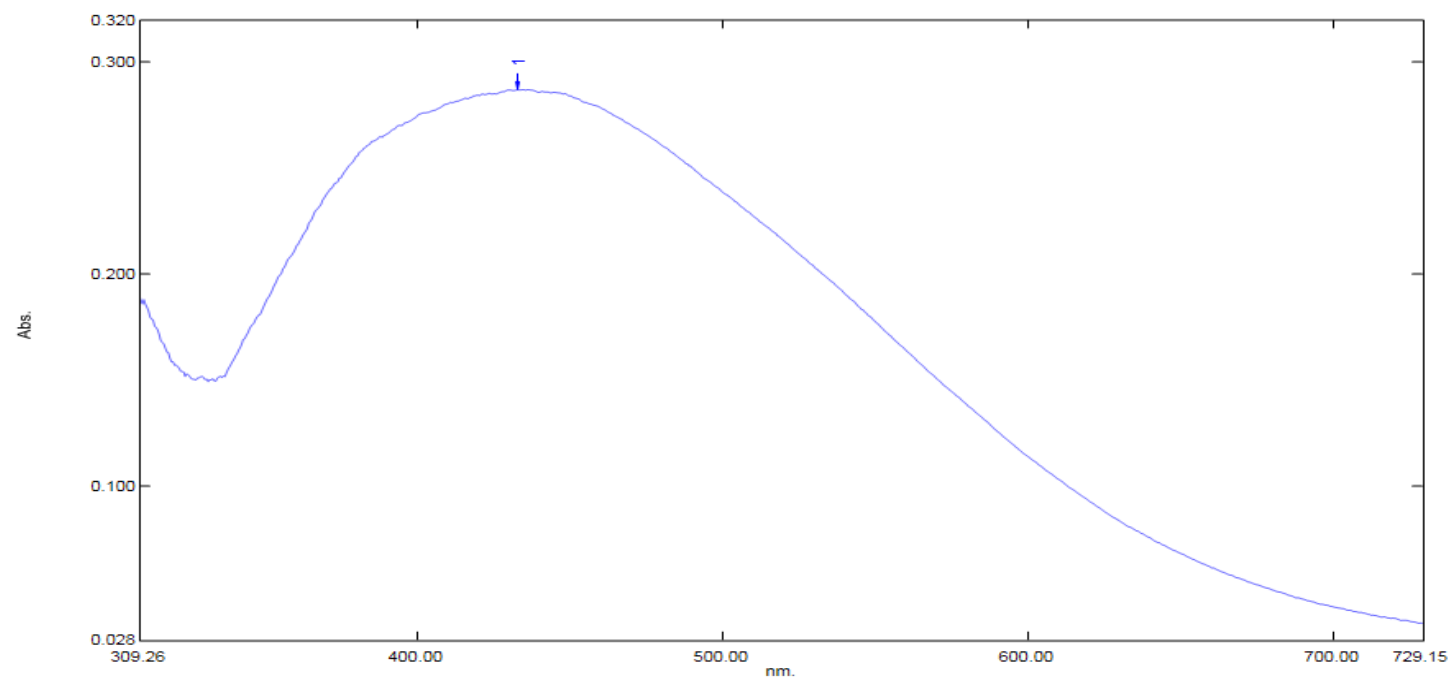

Fig.-1: UV- Visible Spectrum of Silver Nanoparticles

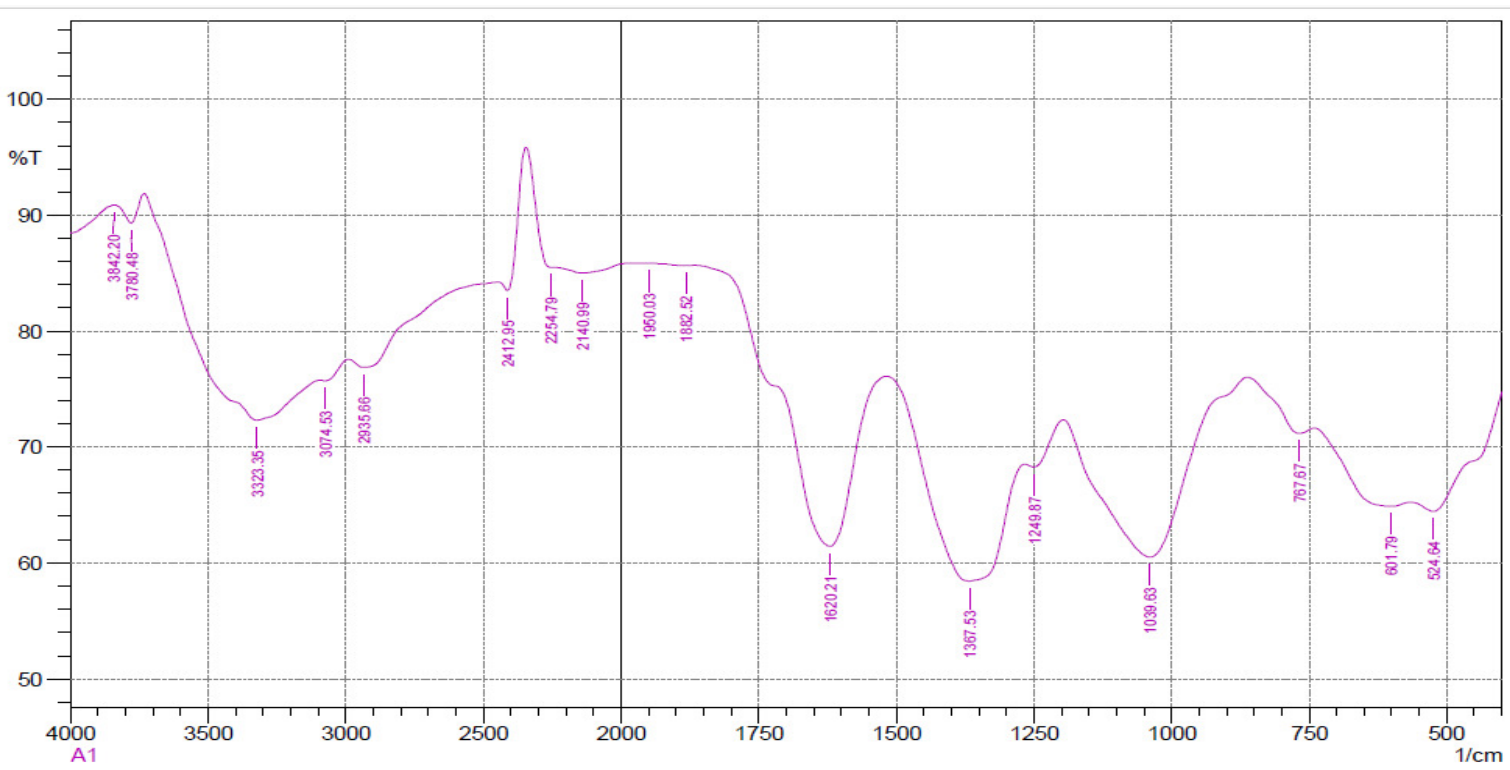

Fig.-2: FT-IR Spectrum of Silver Nanoparticle

\section{X-Ray Diffraction Measurement (PXRD)}

X-ray scattering techniques were a family of non-destructive analytical techniques which reveal information about the crystallographic structure, chemical composition and physical properties of materials. These techniques are based on observing the scattered intensity of an X-Ray beam hitting sample as a function of incident and scattered angle, polarization and wavelength or energy. PXRD was performed at D8 Advanced PXRD, Bruker and the spectra were analyzed using origin 6.0 software. The crystalline domain size was calculated from the width of the PXRD peaks, assuming that they are free from non-uniform strains, using Scherer's formula:

\section{$\mathrm{D}=0.94 \lambda / \beta \cos \theta$}


Where $\mathrm{D}$ is the average crystalline domain size perpendicular to the reflecting planes, $\lambda$ is the X-Ray wavelength, $\beta$ is full width at half maximum (FWHM) and $\theta$ is the diffraction angle. To eliminate additional instrumental broadening FWHM was corrected, using the FWHM from the large grained Si sample.

$$
\beta_{\text {corrected }}=\left(\mathrm{FWHM}^{2} \text { sample }-\mathrm{FWHM}^{2} \mathrm{Si}\right)^{2}
$$

The above-modified formula is valid only when the crystallite size smaller than 100nm. (Cullity,1978)

\section{Scanning Electron Microscopy (SEM) Analysis}

The SEM image represents the formation of silver nanoparticles was depicted in Fig.-2. Also, the grains have aggregated to form nanoclusters.

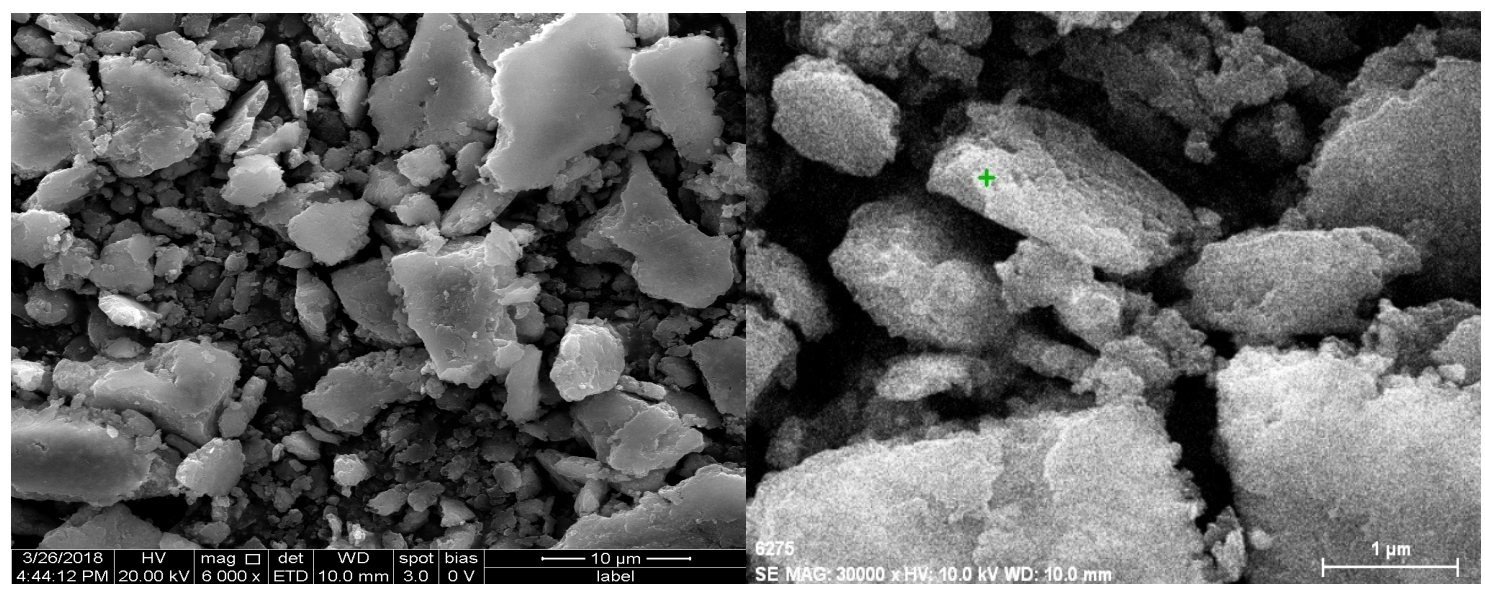

Fig.-3: SEM image of silver Nanoparticles

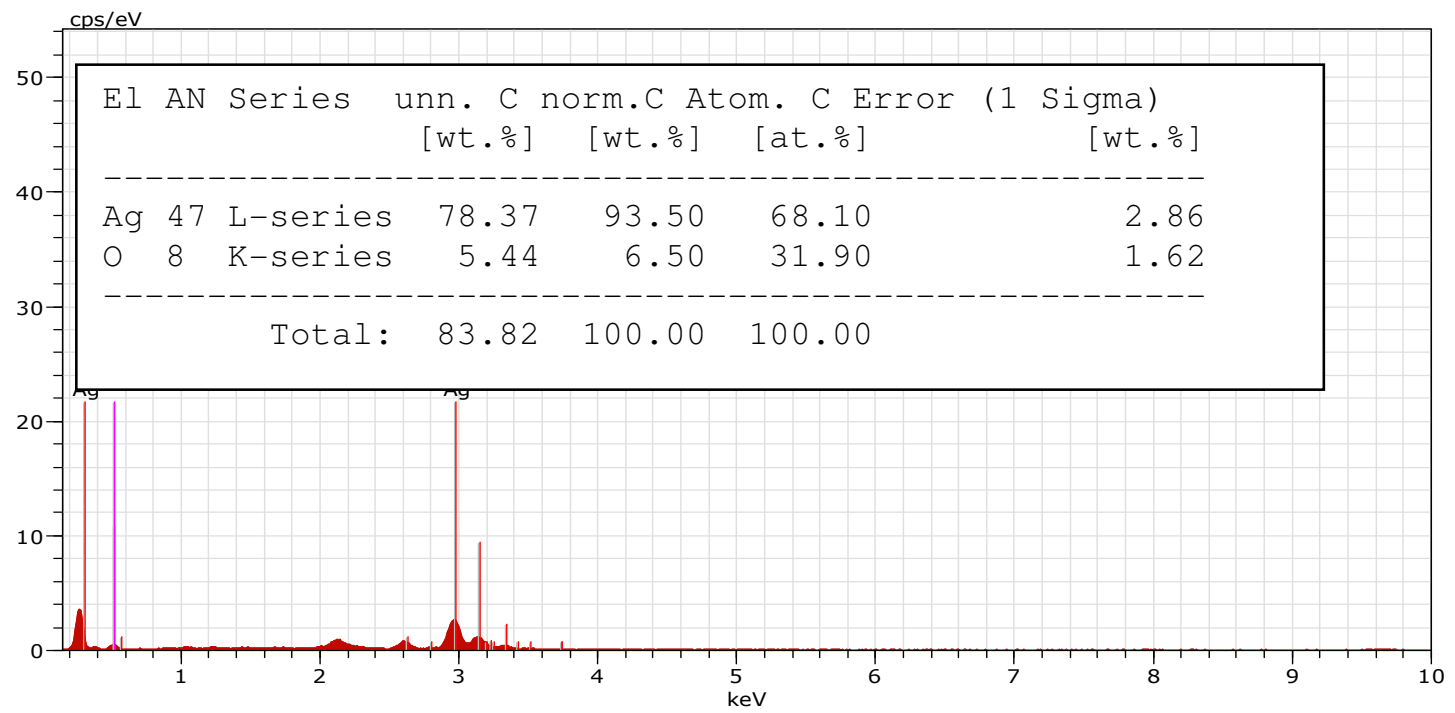

Fig.-4:EDX spectrum of Silver Nanoparticles

\section{Antimicrobial Activity of Silver Nanoparticles}

Antimicrobial action of biosynthesized silver nanoparticles was considered against pathogenic bacteria (clinical isolates) utilizing agar well dispersion strategy and zone of hindrance was delineated in Figure.7 and Table-3. Wells were loaded with diverse centralizations of $250 \mu \mathrm{g} / \mathrm{mL}, 500 \mu \mathrm{g} / \mathrm{mL}$, and $1000 \mu \mathrm{g} / \mathrm{mL}$ of silver nanoparticles individually. Greatest zone of hindrance was watched with Staphylococcusaureus $7 \mathrm{~mm}$ at $50 \mu \mathrm{l}$ of silver nanoparticles fixation and E.coli indicated a great zone of restraint at 
$1000 \mu \mathrm{g} / \mathrm{mL}$. The antimicrobial components of nanosilver particles may contrast from species to types of microbes and size of the nanoparticles.
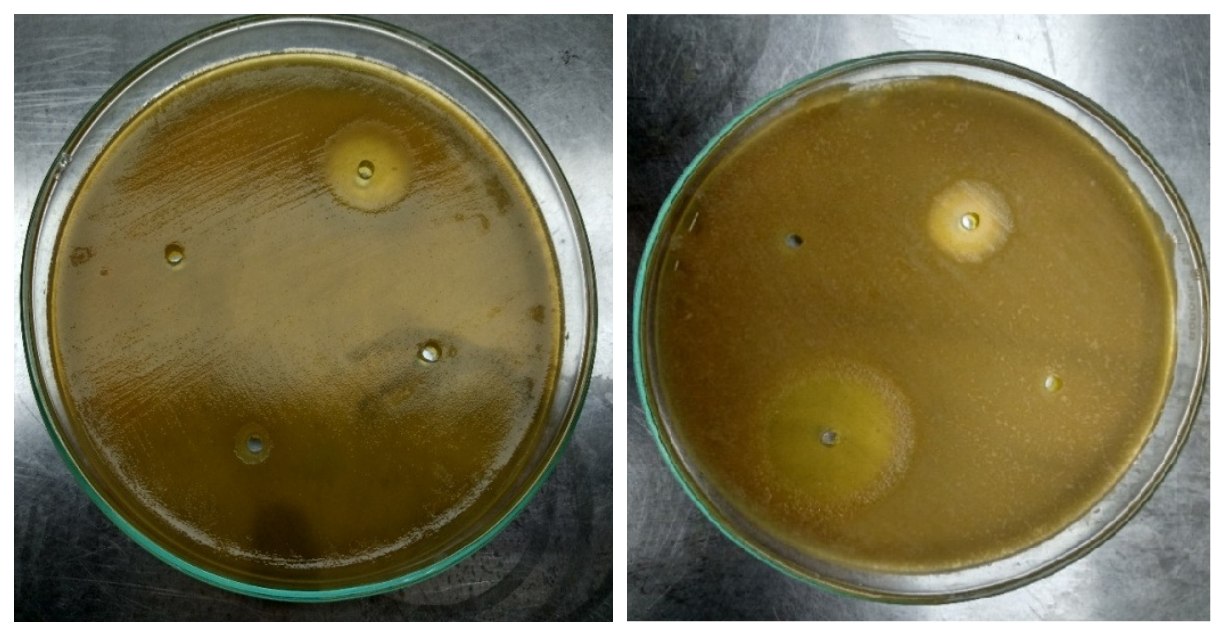

Fig.-5: Antimicrobial Activity of AgNPs (a) Staphylococcus aureus, (b)E.coli

Table-3: Inhibition Zone of AgNPs

\begin{tabular}{c|c|c|c|c}
\hline \multirow{2}{*}{ Organism } & \multicolumn{4}{|c}{ Zone of Inhibition (diameter in mm) } \\
\cline { 2 - 5 } & $1000 \mu \mathrm{g} / \mathrm{mL}$ & $500 \mu \mathrm{g} / \mathrm{mL}$ & $250 \mu \mathrm{g} / \mathrm{mL}$ & Control \\
\hline Staphylococcus aureus & 13 & $\geq 10$ & Nil & Nil \\
\hline Escherichia coli & 28 & 15 & Nil & Nil \\
\hline
\end{tabular}

\section{CONCLUSION}

In the present study, it was found that stem of Mirabilis jalapa could be a green source for the synthesis of silver nanoparticles through a green chemistry approach with several advantages. A simple, cheap, fast and green method to synthesize silver nanoparticles using Mirabilis jalapa. The reduction of the metal ions through the stem extract led to the formation of silver nanoparticles of fairly well-defined dimensions. The synthesized silver nanoparticles were characterized by using UV visible FTIR, Antimicrobial Activity and PXRD. This process was able to synthesize silver nanoparticles within $12 \mathrm{hrs}$ which was confirmed by absorption peak at $449 \mathrm{~nm}$ in UV-Vis spectrum.

\section{REFERENCE}

1. M. Poliakoff and P. Anastas, Nature, 413, 257 (2001).

2. M. Poliakoff, J. M. Fitzpatrick, T. R. Farren and P. T. Anastas, Science, 297, 807 (2002).

3. R. A. Gross and B. Kalra, Science, 297, 803(2002),

4. J. M. Desimone, Science, 297, 799(2002).

5. P. Raveendran, J. Fu and S. L. Wallen, Journalof American Chemical Society, 125, 13940(2003)

6. G.M. Srirangam, K. Parameswara Rao, Rasayan J.Chem, 10, 46 (2017), DOI: 10.7324/RJC.2017.1011548

7. K. Kalimuthu, R.S. Babu, D.Venkataraman, M. Bilal, S. Gurunathan S Colloids Surf. B, 65, 150(2008).

8. G. Reddy, A. Thakur, Rasayan J.Chem, 10, 695(2017), DOI: 10.7324/RJC.2017.1031702

9. U. Klueh, V. Wagner, S. Kelly, A. Johnson, J.D. Bryers, J. Biomed. Mater.Res. 53, 621 (2000).

10. A.Saxena, R.M. Tripathi, R.P. Singh, J. Nanomater. Bios.5, 427 (2010).

11. D.S. Goodsell, Bio nanotechnology: Lessons from Nature. Wiley-Liss, Inc., Hoboken, New Jersey. p.1-347(2004).

12. C. T.Handoko, A. Huda1, M. D.Bustan, B. Yudono, F. Gulo, Rasayan J.Chem, 10, 1137(2017), DOI: 10.7324/RJC.2017.1041875

13. R. Mahendra, Y. Alka, G. Aniket, Biotechnol Adv.,27,76 (2009).

[RJC-5136/2018] 